\title{
Study of Geiger-mode APDs performances at cryogenic temperatures
}

\section{A. Bondar}

Budker Institute of Nuclear Physics, 11 Lavrentiev avenue, Novosibirsk, 630090 Russia

Novosibirsk State University, 2 Pirogova street, Novosibirsk, 630090 Russia

\section{A. Buzulutskov}

Budker Institute of Nuclear Physics, 11 Lavrentiev avenue, Novosibirsk, 630090 Russia Novosibirsk State University, 2 Pirogova street, Novosibirsk, 630090 Russia

\section{A. Dolgov}

Novosibirsk State University, 2 Pirogova street, Novosibirsk, 630090 Russia

\section{E. Shemyakina}

Budker Institute of Nuclear Physics, 11 Lavrentiev avenue, Novosibirsk, 630090 Russia Novosibirsk State University, 2 Pirogova street, Novosibirsk, 630090 Russia

\section{A. Sokolov ${ }^{1}$}

Budker Institute of Nuclear Physics, 11 Lavrentiev avenue, Novosibirsk, 630090 Russia Novosibirsk State University, 2 Pirogova street, Novosibirsk, 630090 Russia

E-mail: A.V.Sokoloveinp.nsk.su

\begin{abstract}
We compare the performances of different types of Geiger-mode APDs (GAPDs or SiPMs), manufactured by CPTA Company, at cryogenic temperature, namely at $87 \mathrm{~K}$. Noise rate, gain characteristics and signal shapes were measured, both at room and cryogenic temperature. Four types of GAPDs were investigated, having an active area of 1.0, 2.1x2.1, 2.5x2.5, and $3 \times 3 \mathrm{~mm}^{2}$. The best performance showed the GAPD with $2.1 \times 2.1 \mathrm{~mm}^{2}$ active area, in terms of the maximum gain, minimum noise rate and effective active area. This study is done in the frame of the project, developed in our laboratory, on the Cryogenic Avalanche Detector (CRAD) with optical readout using combined THGEM/GAPD-matrix multiplier.
\end{abstract}

International Workshop on New Photon-detectors,

LAL Orsay, France

June 13-15, 2012

$1 \quad$ Speaker and corresponding author 


\section{Introduction}

In our laboratory we develop Cryogenic Avalanche Detectors (CRADs) with optical readout using combined THGEM/GAPD multiplier [1],[2]. Such detectors may have an ultimate sensitivity, namely single electron sensitivity, at reduced noises which is of primary importance for coherent neutrino-nucleus scattering and dark matter search experiments [1]. To achieve these goals, the detector must have the lowest possible noise level at reasonable gains. In connection with this, the study of noise and gain characteristics of GAPDs of different types at cryogenic temperatures has been conducted.

\section{Experimental setup}

The experimental setup is shown in Figure 1: it was similar to that used in our previous works [3],[4]. Several GAPD assemblies were installed inside a 9 litre cryogenic chamber filled with Ar. The temperature inside the chamber might be varied between $86 \mathrm{~K}$ and $295 \mathrm{~K}$. The GAPD assemblies were consisted of GAPDs of different types produced by the CPTA company [5]: these included CPTA types of 143-32, 149-35, 150-50 and 140-40, having an active area of $1.0,2.1 \times 2.1,2.5 \times 2.5$ and $3 \times 3 \mathrm{~mm}^{2}$ respectively. The signals from GAPDs were read out via a 1 $\mathrm{m}$ long twisted pair cables connected to a fast amplifier (CPTA [5]) with $300 \mathrm{MHz}$ bandwidth and amplification factor of 30. A TDS5032B digital oscilloscope serves as a data acquisition system. When studying the GAPD characteristics, the THGEM multiplier shown in the figure was inactive. Further details of the experimental setup and measurement procedures can be found elsewhere [3],[4],[6].
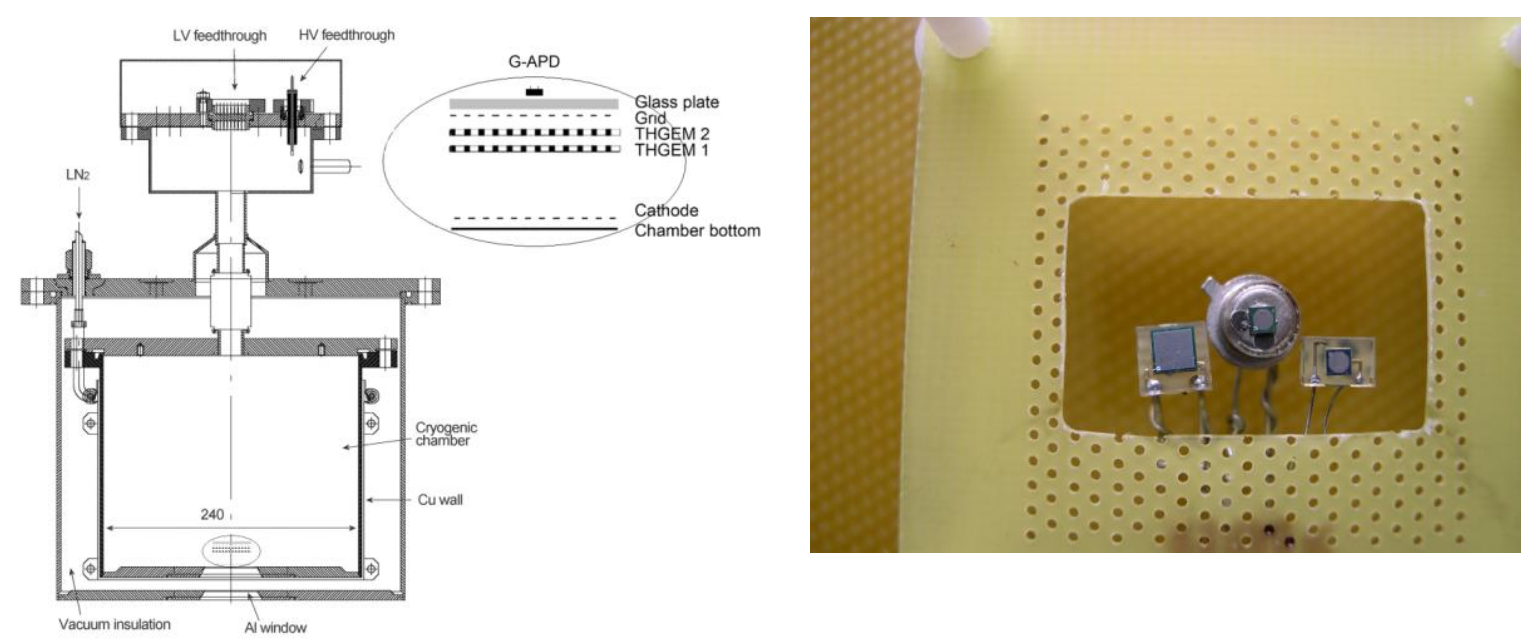

Figure 1. Schematic view of the experimental setup to study GAPDs performances at cryogenic temperatures (left) and the photograph of one of the GAPD assemblies installed inside the cryogenic chamber (right). 


\section{Experimental results}

In Figure 2 typical the pulse shapes of the noise signals from different GAPDs at $87 \mathrm{~K}$ are shown. One can see that single-, double- and triple-pixel signals are well identified. The GAPD gain was measured using the noise signals. The appropriate experimental procedure was the following: the area of the single-pixel noise pulse was determined by means of the oscilloscope. Then, knowing the fast amplifier gain, the single-pixel charge was calculated; this charge is equal to the GAPD gain at a given bias voltage.

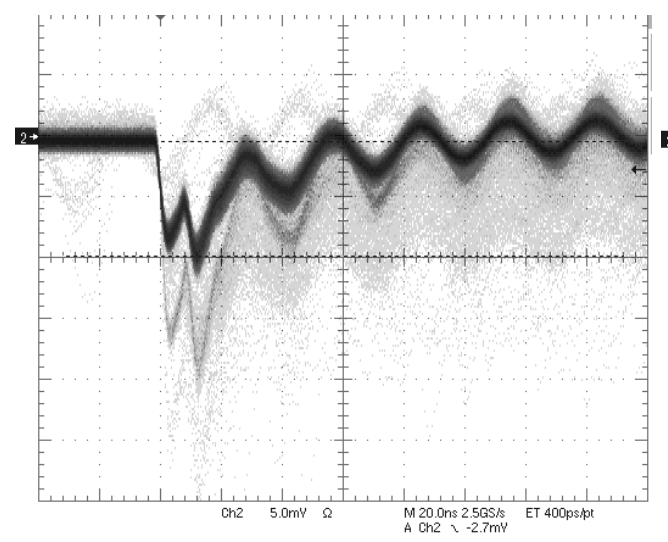

a)

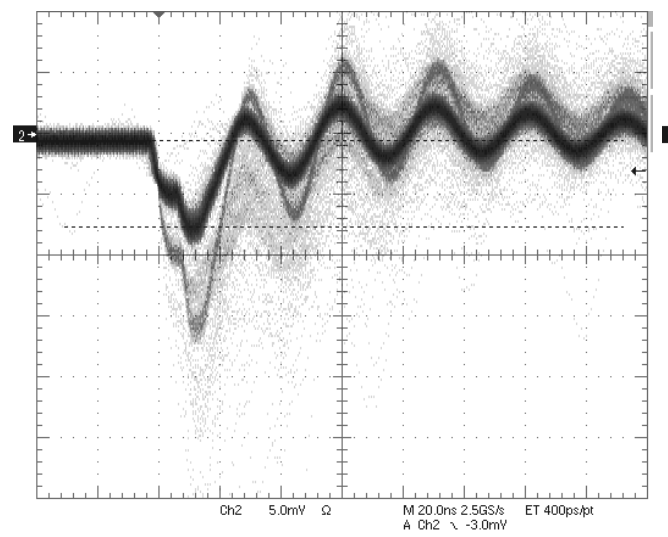

c)

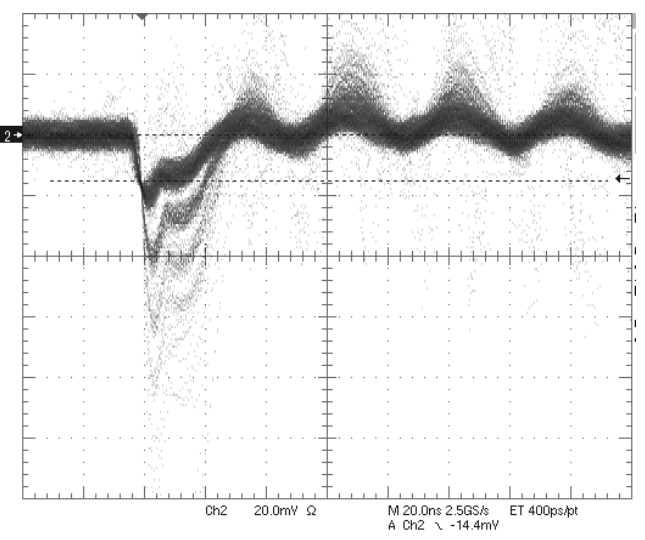

b)

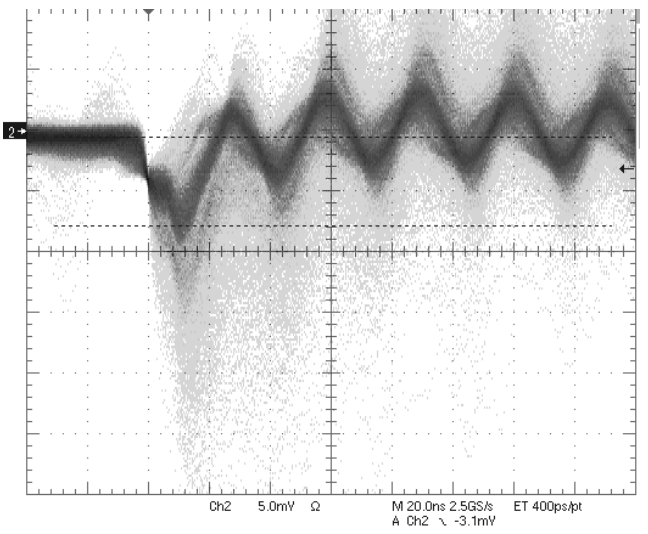

d)

Figure 2. Typical noise signals from GAPDs of different active areas: a) $1.0 \mathrm{~mm}^{2}$ at $295 \mathrm{~K}$ and $35 \mathrm{~V}$; b) $2.1 \times 2.1 \mathrm{~mm}^{2}$ at $87 \mathrm{~K}$ and $46 \mathrm{~V}$; c) $2.5 \times 2.5 \mathrm{~mm}^{2}$ at $87 \mathrm{~K}$ and $50 \mathrm{~V}$; d) $3 \times 3 \mathrm{~mm}^{2}$ at $87 \mathrm{~K}$ and $44 \mathrm{~V}$.

Figure 3 shows gain-voltage characteristics of the GAPDs studied, namely the single pixel charge as a function of the bias voltage at a cryogenic temperature of $87 \mathrm{~K}$ and at room temperature. One can see that the maximum pixel amplitude at the cryogenic temperature is substantially higher compared to that at room temperature, confirming the superior performance of GAPDs at cryogenic temperatures [3],[6]. 

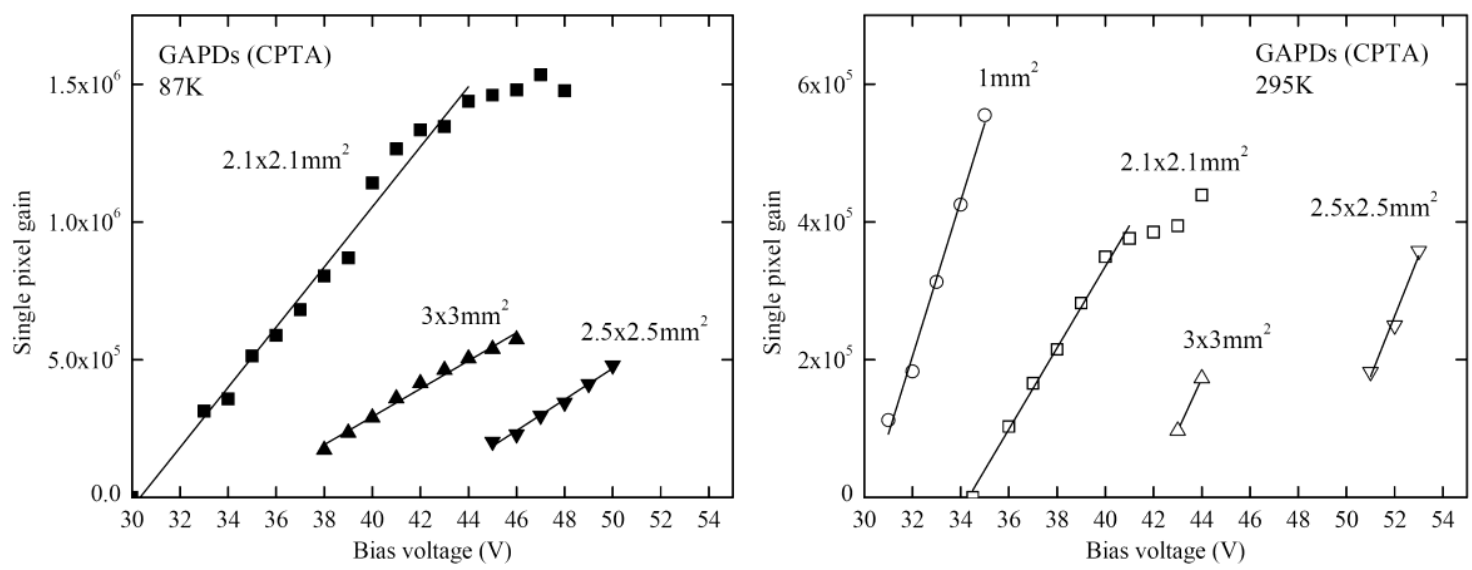

Figure 3. Gain characteristics of different GAPD types at temperatures of $87 K$ and $295 K$.

In Figure 4 one can see the significant difference in terms of the noise rate between the GAPD performance at room and cryogenic temperatures: for the latter the noise rate is considerably reduced, again confirming the superior performance of GAPDs at cryogenic temperatures. The noise rate was measured by counting the noise pulses in a given time interval, the noise signals being recognized by their characteristic pulse-shapes (Figure 2).

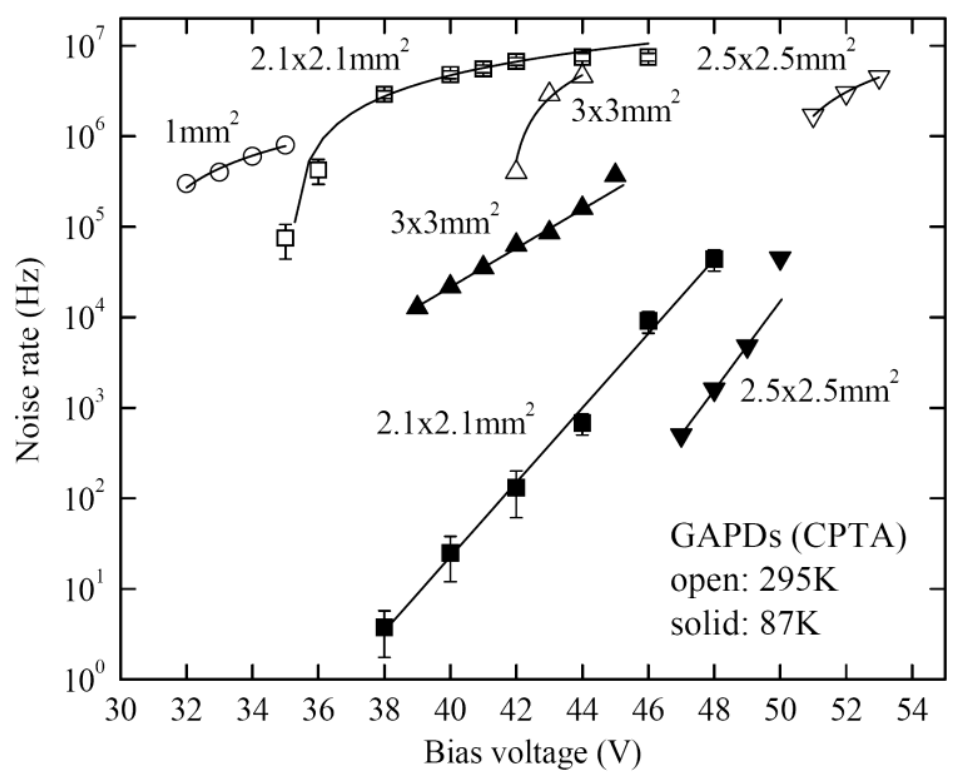

Figure 4. Noise rate for GAPDs of different active areas as a function of the bias voltage at temperatures of $87 \mathrm{~K}$ and $295 \mathrm{~K}$.

Comparing Figs. Figure 3 and Figure 4, one may conclude that the GAPD of the "CPTA 149-35" type, having a reasonable active area, of $2.1 \times 2.1 \mathrm{~mm}^{2}$, has a superior performance in terms of the maximum gain and minimum noise rate. In particular at an overvoltage of $14 \mathrm{~V}$ its gain reached a value of $1.5 \times 10^{6}$, which is a factor of 3 higher than the maximum gains obtained for GAPD types of larger active area. In addition, its noise rate at this maximum gain, $<1 \mathrm{kHz}$, 
is an order and two orders of magnitude lower compared to the GAPD types with an active area of $2.5 \times 2.5$ and $3 \times 3 \mathrm{~mm}^{2}$ respectively (at their maximum gains).

\section{Conclusions}

GAPDs of different types show rather diverse behaviour at cryogenic temperatures. The GAPD of the "CPTA 149-35" type with an active area of $2.1 \times 2.1 \mathrm{~mm}^{2}$ showed a superior performance in terms of the maximum gain and minimum noise rate, at $87 \mathrm{~K}$. Compared to other GAPD types that have higher active area, it has a factor of 3 higher maximum gains at more than an order of magnitude lower noise rates.

This work was supported in part by the Ministry of Education and Science of Russian Federation and Grants of the Government of Russian Federation (11.G34.31.0047) and the Russian Foundation for Basic Research (12-02-91509-CERN_a and 12-02-12133-ofi_m).

\section{References}

[1] A. Buzulutskov, Advances in Cryogenic Avalanche Detectors, JINST 7 (2012) C02025.

[2] A. Bondar et al., Study of infrared scintillations in gaseous and liquid argon. Part II: light yield and possible applications, JINST 7 (2012) P060014.

[3] A. Bondar et al., Direct observation of avalanche scintillations in a THGEM-based twophase Ar avalanche detector using Geiger-mode APD, JINST 5 (2010) P08002.

[4] A. Bondar et al, On the low-temperature performances of THGEM and THGEM/GAPD multipliers in gaseous and two-phase Xe, JINST 6 (2011) P07008.

[5] CPTA company, 〈http://www.cpta-apd.ru>.

[6] A. Bondar et al., Geiger mode APD performance in a cryogenic two-phase Ar avalanche detector based on THGEMs, NIM A 628 (2011) 364. 